PACS 31.15.A-; 32.30.-r

Chernyshev A. S., Efimova E. A., Buyadzhi V. V., Nikola L. V.

I.I. Mechnikov Odesa National University, Dvoryanskaya str. , 2, Odesa, 65000

Odesa State Environmental University, 15, Lvovskaya str., Odesa-16, 65016

E-mail: buyadzhivv@gmail.com

\title{
CASCADE OF AUGER TRANSITIONS IN SPECTRUM OF XENON: THEORETICAL DATA
}

\begin{abstract}
The energy parameters of the Auger transitions for the xenon atomic system are calculated within the combined relativistic energy approach and relativistic many-body perturbation theory with the zeroth order density functional approximation. The results are compared with reported experimental data as well as with those obtained by semiempirical method. The important point is linked with an accurate accounting for the complex exchange-correlation (polarization) effect contributions and using the optimized one-quasiparticle representation in the relativistic many-body perturbation theory zeroth order that significantly provides a physically reasonable agreement between theory and experiment.
\end{abstract}

\section{Introduction}

Development of the novel experimental methods in a modern Auger- spectroscopy allows to open new interesting features especially for the heavy complex atomic systems. It is easily to understand that a synchrotron radiation sources provide an effective way to ionize deep core hole States in heavy multielectron systems (e.g. [1]). As result, new advanced data on the energy and spectral parameters of the complex atomic systems can be obtained.

Let us remind following to ref . [2] that studies in modern atomic physics (spectroscopy, spectral lines theory, theory of atomic collisions etc), astrophysics, plasma physics, laser physics and quantum and photoelectronics requires an availability of sets of correct data on the energetic, spectroscopic and structural properties of atoms, including the data on the Auger transitions [1-8]. The kinetic energy of the ejected Auger electron is measured by Auger-electron spectroscopy (AES). Sensing the Auger spectra in atomic systems and solids gives the important data for the whole number of scientific and technological applications. So called two-step model is used most widely when calculating the Auger decay characteristics [1-5]. Since the vacancy lifetime in an inner atomic shell is rather long (about $10^{-17}$ to $10^{-14} \mathrm{~s}$ ), the atom ionization and the Auger emission are con- sidered to be two independent processes. In the more correct dynamic theory of the Auger effect $[2,3]$ the processes are not believed to be independent from one another. The fact is taken into account that the relaxation processes due to Coulomb interaction between electrons and resulting in the electron distribution in the vacancy field have no time to be over prior to the transition.

In fact, a consistent Auger decay theory has to take into account correctly a number of correlation effects, including the energy dependence of the vacancy mass operator, the continuum pressure, spreading of the initial state over a set of configurations etc [119]. The most widespread theoretical studying the Auger spectra parameters is based on using the multi-configuration Dirac-Fock (MCDF) calculation [2,3]. The theoretical predictions based on MCDF calculations have been carried out within different approximations and remained hitherto nonsatisfactory in many relations. Earlier [8-13] it has been proposed relativistic perturbation theory (PT) method of the Auger decay characteristics for complex atoms, which is based on the Gell-Mann and Low S-matrix formalism energy approach) and QED PT formalism [4-7]. The novel element consists in using the optimal basis of the electron state functions derived from the minimization condition for the calibration-non-invariant 
contribution (the second order PT polarization diagrams contribution) to the imaginary part of the multi-electron system energy already at the first non-disappearing approximation of the PT. Earlier it has been applied in studying the Auger decay characteristics for a set of neutral atoms, quasi-molecules and solids. Besides, the ionization crosssections of inner shells in various atoms and the Auger electron energies in solids were estimated. Here we apply the combined relativistic energy approach and relativistic many-body perturbation theory with the zeroth order density functional approximation is applied to determination of the energy parameters of the Auger transitions of the xenon atomic system.

\section{The theoretical method}

In Refs. [2, 8-17] the fundamentals of the relativistic many-body PT formalism have been in detail presented, so further we are limited only by the key elements, following the cited Refs. Let us remind that the majority of complex atomic systems possess a dense energy spectrum of interacting states. In Refs. [2-13, 19-38] there is realized field procedure for calculating the energy shifts $\Delta \mathrm{E}$ of degenerate states, which is connected with the secular matrix $M$ diagonalization. The whole calculation of the energies and decay probabilities of a non-degenerate excited state is reduced to the calculation and diagonalization of the $M$. The complex secular matrix $M$ is represented in the form $[2,9,10]$ :

$$
M=M^{(0)}+M^{(1)}+M^{(2)}+M^{(3)} .
$$

where $M^{(0)}$ is the contribution of the vacuum diagrams of all order of PT, and $M^{(1)}$, $M^{(2)}, M^{(3)}$ those of the one-, two- and threeQP diagrams respectively. The diagonal matrix $M^{(1)}$ can be presented as a sum of the independent 1QP contributions. The optimized 1-QP representation is the best one to determine the zeroth approximation. In the relativistic energy approach [4-9], which has received a great applications during solving numerous problems of atomic, molecular and nuclear physics (e.g., see Refs. [10-13]), the imaginary part of electron energy shift of an atom is directly connected with the radiation decay possibility (transition probability). An approach, using the Gell-Mann and Low formula with the QED scattering matrix, is used in treating the relativistic atom. The total energy shift of the state is usually presented in the form:

$$
\Delta \mathrm{E}=\operatorname{Re} \Delta \mathrm{E}+\mathrm{i} \Gamma / 2
$$

where $\Gamma$ is interpreted as the level width, and the decay possibility $\mathrm{P}=\Gamma$. The imaginary part of electron energy of the system, which is defined in the lowest order of perturbation theory as [4]:

$$
\operatorname{Im} \Delta E(B)=-\frac{e^{2}}{4 \pi} \sum_{\substack{\alpha>n>f \\ \alpha<n \leq f]}}^{\left|\omega_{\alpha n \alpha n}\right|},
$$

where $(\alpha>n>f)$ for electron and $(\alpha<n<f)$ for vacancy. Under calculating the matrix elements (3) one should use the angle symmetry of the task and write the expansion for potential $\sin |\omega| \mathrm{r}_{12} / \mathrm{r}_{12}$ on spherical functions as follows [4]:

$$
\begin{array}{r}
\frac{\sin |\omega| r_{12}}{r_{12}}=\frac{\pi}{2 \sqrt{r_{1} r_{2}}} \sum_{\lambda=0}^{\infty}(\lambda) J_{\lambda+1 / 2}\left(|\omega| r_{1}\right) . \\
. J_{\lambda+1 / 2}\left(|\omega| r_{1}\right) J_{\lambda+1 / 2}\left(|\omega| r_{2}\right) P_{\lambda}\left(\cos r_{1} r_{2}\right)
\end{array}
$$

where $J$ is the Bessel function of first kind and $(\lambda)=2 \lambda+1$. This expansion is corresponding to usual multipole one for probability of radiative decay.

Within the frame of QED PT approach the Auger transition probability and the Auger line intensity are defined by the square of an electron interaction matrix element having the form [4]:

$V_{1234}^{\omega}=\left[\left(j_{1}\right)\left(j_{2}\right)\left(j_{3}\right)\left(j_{4}\right)\right]^{1 / 2} \sum_{\lambda \mu}(-1)^{\mu}\left(\begin{array}{cc}j_{1} j_{3} & \lambda \\ m_{1}-m_{3} & \mu\end{array}\right) \times \operatorname{Re} Q_{\lambda}(1234)^{\prime}$ 


$$
Q_{\lambda}=Q_{\lambda}^{\mathrm{Qul}}+Q_{\lambda}^{\mathrm{Br}}
$$

The terms $Q_{\lambda}^{\mathrm{Qul}}$ and $Q_{\lambda}^{\mathrm{Br}}$ correspond to subdivision of the potential into Coulomb part $\cos |\omega| r_{12} / r_{12}$ and Breat one, $\cos |\omega| r_{12} \alpha_{1} \alpha_{2} / r_{12}$. The real part of the electron interaction matrix element is determined using expansion in terms of Bessel functions:

$\frac{\cos |\omega| r_{12}}{\eta_{2}}=\frac{\pi}{2 \sqrt{\eta_{1} r_{2}}} \sum_{\lambda=0}(\lambda) J_{\lambda+1 / 2}\left(|\omega| r_{<}\right) J_{-\lambda-1 / 2}\left(|\omega| r_{>}\right) P_{\lambda}\left(\cos \boldsymbol{r}_{1} \mathbf{r}_{2}\right)$

where $J$ is the $1^{\text {st }}$ order Bessel function, $(\lambda)=2 \lambda+1$.

The Coulomb part $Q_{\lambda}^{\text {Qul }}$ is expressed in terms of radial integrals $R_{\lambda}$, angular coefficients $S_{\lambda}$ [4]:

$$
\begin{aligned}
& \operatorname{Re} Q_{\lambda}^{\mathrm{Qul}}=\frac{1}{Z} \operatorname{Re}\left\{R_{l}(1243) S_{\lambda}(1243)+R_{\lambda}(\widetilde{1} 24 \widetilde{3}) S_{\lambda}(\widetilde{1} 24 \widetilde{3})+\right. \\
& \left.+R_{\lambda}(1 \widetilde{2} \widetilde{4} 3) S_{\lambda}(1 \widetilde{2} \widetilde{4} 3)+R_{\lambda}(\widetilde{1} \widetilde{2} \widetilde{4} \widetilde{3}) S_{\lambda}(\widetilde{1} \widetilde{2} \widetilde{4} \widetilde{3})\right\} .
\end{aligned}
$$

As a result, the Auger decay probability is expressed in terms of $\operatorname{Re} Q_{\lambda}(1243)$ matrix elements:

$$
\operatorname{Re} R_{\lambda}(1243)=\iint d \eta r_{1}^{2} r_{2}^{2} f_{1}\left(\eta_{1}\right) f_{3}\left(\eta_{1}\right) f_{2}\left(r_{2}\right) f_{4}\left(r_{2}\right) Z_{\lambda}^{(1)}\left(r_{<}\right) Z_{\lambda}^{(1)}\left(r_{>}\right)
$$

where $f$ is the large component of radial part of single electron state Dirac function; function $Z$ and angular coefficient are defined in Refs. [4-7]. The other items in (7) include small components of the Dirac functions; the sign « means that in (7) the large radial component $f_{i}$ is to be changed by the small $g_{i}$ one and the moment $l_{i}$ is to be changed by $\tilde{l}_{i}=l_{i}-1$ for Dirac number $æ_{1}>0$ and $l_{i}+1$ for $æ_{i}<0$. The Breat part of $Q$ is defined in $[4,11]$. The Auger width is obtained from the adiabatic Gell-Mann and Low formula for the energy shift [4].

The direct contribution to the Auger level width with a vacancy $n_{\alpha} l_{\alpha} j_{\alpha} m_{\alpha}$ is as follows:

$$
\sum_{\lambda} \frac{2}{(\lambda)\left(j_{\alpha}\right)} \sum_{\beta \gamma \leq f} \sum_{k>f} Q_{\lambda}(\alpha k \gamma \beta) Q_{\lambda}(\beta \gamma k \alpha),
$$

while the exchange diagram contribution is:

$$
\frac{2}{\left(j_{\alpha}\right)} \sum_{\lambda_{1} \lambda_{2} \beta \gamma \leq f k>f} \sum_{\lambda_{1}}(\alpha k \gamma \beta) Q_{\lambda_{2}}(\beta \gamma k \alpha)\left\{\begin{array}{lll}
j_{\alpha} & j_{\gamma} & \lambda_{2} \\
j_{k} & j_{\beta} & \lambda_{1}
\end{array}\right\} .
$$

The partial items of the $\sum_{\beta \gamma} \sum_{k} \widehat{\text { sum an- }}$ swer to contributions of $\alpha^{-1} \rightarrow(\beta \gamma)^{-1} K$ channels resulting in formation of two new vacancies $\beta \gamma$ and one free electron $k$ : $\omega_{k}=\omega_{\alpha}+\omega_{\beta}-\omega_{\alpha}$.

The calculating of all matrix elements, wave functions, Bessel functions etc is reduced to solving the system of differential equations. The formulas for the autoionization (Auger) decay probability include the radial integrals $R_{\alpha}(\alpha k \gamma \beta)$, where one of the functions describes electron in the continuum state.

When calculating this integral, the correct normalization of the wave functions is very important, namely, they should have the following asymptotic at $r \rightarrow 0$ :

$$
\left.\begin{array}{l}
f \\
g
\end{array}\right\} \rightarrow(\lambda \omega)^{-1 / 2}\left\{\begin{array}{l}
{\left[\omega+(\alpha Z)^{-2}\right]^{-1 / 2} \sin (k r+\delta),} \\
{\left[\omega-(\alpha Z)^{-2}\right]^{-1 / 2} \cos (k r+\delta) .}
\end{array}\right.
$$

The important aspect of the whole procedure is an accurate accounting for the exchange-correlation effects. We have used the generalized relativistic Kohn-Sham density functional [8-17] in the zeroth approximation of relativistic PT; naturally, the perturbation operator contents the operator (7) minus the cited Kohn-Sham density functional. Further the wave functions are corrected by accounting of the first order PT contribution. Besides, we realize the procedure of optimization of relativistic orbitals base. The main idea is based on using ab initio optimization procedure, which is reduced to minimization of the gauge dependent multielectron contribution $\operatorname{Im} \Delta E_{\text {ninv }}$ of the lowest QED PT corrections to the radiation widths of atomic levels. 
The formulae for the Auger decay probability include the radial integrals $R_{\alpha}(\alpha k \gamma \beta)$, where one of the functions describes electron in the continuum state. The energy of an electron formed due to a transition $j k l$ is defined by the difference between energies of atom with a hole at $j$ level and doubleionized atom at $k l$ levels in final state:

$$
E_{A}\left(j k l,{ }^{2 S+1} L_{J}\right)=E_{A}^{+}(j)-E_{A}^{2+}\left(k l,{ }^{2 S+1} L_{J}\right)
$$

To single out the above-mentioned correlation effects, the equation (13) can be presented as $[8,9]$ :

$$
E_{A}\left(j k l,{ }^{2 S+1} L_{J}\right)=E(j)-E(k)-E(l)-\Delta\left(k, l ;{ }^{2 S+1} L_{J}\right),
$$

where the item $\Delta$ takes into account the dynamic correlation effects (relaxation due to hole screening with electrons etc.) To take these effects into account, the set of procedures elaborated in the atomic theory [8-13] is used. All calculations are performed on the basis of the modified numeral code Superatom (version 93).

\section{Results and conclusion}

Below the energy parameters of the Auger transitions for the xenon are presented and compared with the experimental data as well as with those obtained by semiempirical and perturbation theory methods. In table 1 we present data on the initial and final states of the most intense $4 \mathrm{~d}^{-2} \rightarrow 4 \mathrm{~d}^{-1} \rightarrow 5 \mathrm{p}^{-2}$ and $4 \mathrm{~d}^{-1} 5 \mathrm{p}^{-2} \rightarrow 5 \mathrm{p}^{-4}$ Auger transitions in the neutral xenon.

Our theoretical data (the relativistic manybody PT) and the theoretical semiempirical data by Jonauskas et al [1], and relativistic perturbation theory data [9] for the energies of the Auger transitions are also given.

The analysis of the presented results in table 1 results in the conclusions that the précised description of the Auger processes requires the detailed accurate accounting for the exchange-correlation effects, including the particle-hole interaction, screening effects and iterations of the mass operator.
Table 1. Calculated and experimental energies of the Auger transitions $\mathrm{E}_{\mathrm{k}}$ for $\mathrm{Xe}$

\begin{tabular}{|c|c|c|c|c|c|}
\hline $\begin{array}{l}\text { Initial } \\
\text { state }\end{array}$ & Final state & $\begin{array}{l}\mathrm{E}_{\text {th }} \\
{[1]}\end{array}$ & $\begin{array}{l}\mathrm{E}_{\mathrm{th}}, \\
{[9]}\end{array}$ & $\begin{array}{l}\mathrm{E}_{\text {th }}, \\
\text { our }\end{array}$ & $\begin{array}{l}\mathrm{E}_{\mathrm{ex}} \\
{[1]}\end{array}$ \\
\hline $\begin{array}{l}{ }^{4 d^{-2}} \\
{ }^{1} G_{4}\end{array}$ & $\begin{array}{l}4 d^{-1} 5 p^{-2}\left({ }^{1} D\right) \\
{ }^{2} F_{5 / 2}\end{array}$ & 31.5 & 31.3 & 31.2 & \\
\hline $\begin{array}{l}4 d^{-2} \\
{ }^{1} G_{4}\end{array}$ & $\begin{array}{l}4 d^{-1} 5 p^{-2}\left({ }^{3} P\right) \\
{ }^{2} F_{7 / 2}\end{array}$ & 31.2 & 31.0 & 30.9 & 30.8 \\
\hline $\begin{array}{l}{ }^{4 d^{-2}} \\
{ }^{1} G_{4}\end{array}$ & $\begin{array}{l}4 d^{-1} 5 p^{-2}\left({ }^{1} D\right) \\
{ }^{2} G_{9 / 2}\end{array}$ & 30.9 & 30.6 & 30.5 & \\
\hline $\begin{array}{l}{ }^{4 d^{-2}} \\
{ }^{1} D^{2}\end{array}$ & $\begin{array}{l}4 d^{-1} 5 p^{-2}\left({ }^{3} P\right) \\
{ }^{2} D_{5 / 2}\end{array}$ & 30.5 & 30.3 & 30.2 & 30.3 \\
\hline $\begin{array}{l}{ }_{4 d^{-2}} \\
{ }^{1} G_{4}\end{array}$ & $\begin{array}{l}4 d^{-1} 5 p^{-2}\left({ }^{3} \mathrm{P}\right) \\
{ }^{2} \mathrm{D}_{3 / 2}\end{array}$ & 30.1 & 30.0 & 29.9 & 29.9 \\
\hline $\begin{array}{l}{ }^{4 d^{-2}} \\
{ }^{1} G_{4}\end{array}$ & $\begin{array}{l}4 d^{-1} 5 p^{-2}\left({ }^{1} D\right) \\
{ }^{2} D_{5 / 2}\end{array}$ & 29.3 & 29.2 & 29.1 & \\
\hline $\begin{array}{l}4^{4} \\
{ }^{-2} \\
{ }^{1} D_{2}\end{array}$ & $4 d^{-1} 5 p^{-2}\left({ }^{1} D\right)$ & 29.1 & 29.1 & 29.0 & 29.1 \\
\hline $\begin{array}{l}{ }^{4 d^{-2}} \\
{ }^{1} G_{4}\end{array}$ & $\begin{array}{l}4 d^{-1} 5 p^{-2}\left({ }^{1} D\right) \\
{ }^{2} F_{7 / 2}\end{array}$ & 29.1 & 29.1 & 29.1 & \\
\hline $\begin{array}{l}4_{d^{-2}}^{-2} \\
{ }^{3} F_{4}\end{array}$ & $\begin{array}{l}4 d^{-1} 5 p^{-2}\left({ }^{3} \mathrm{P}\right) \\
{ }^{4} \mathrm{~F}_{9 / 2}\end{array}$ & 29.1 & 29.1 & 29.1 & \\
\hline $\begin{array}{l}4 d^{-2} \\
{ }^{3} F_{4}\end{array}$ & ${ }^{4} \mathrm{~d}^{-1} 5 \mathrm{p}^{-2}\left({ }^{3} \mathrm{P}\right)$ & 28.4 & 28.3 & 28.2 & \\
\hline $\begin{array}{l}4 d^{-2} \\
{ }^{3} F_{4}\end{array}$ & $\begin{array}{l}4 d^{-1} 5 p^{-2}\left({ }^{3} P\right) \\
{ }^{2} F_{5 / 2}\end{array}$ & 28.1 & 28.0 & 28.2 & 28.3 \\
\hline $\begin{array}{l}{ }^{4 d^{-2}} \\
{ }^{1} G_{4}\end{array}$ & $\begin{array}{l}4 d^{-1} 5 p^{-2}\left({ }^{1} S\right) \\
{ }^{2} D_{5 / 2}\end{array}$ & 27.8 & 27.8 & 27.8 & 27.9 \\
\hline $\begin{array}{l}4^{4} d^{-2} \\
{ }^{3} F_{2}\end{array}$ & $4 d^{-1} 5 p^{-2}\left({ }^{1} D\right)$ & 27.4 & 27.3 & 27.2 & 27.1 \\
\hline $\begin{array}{l}4 \mathrm{~d}^{-2} \\
{ }^{3} \mathrm{P}_{1}\end{array}$ & $\begin{array}{l}4 d^{-1} 5 p^{-2}\left({ }^{1} S\right) \\
{ }^{2} D_{5 / 2}\end{array}$ & 27.0 & 26.9 & 26.8 & \\
\hline $\begin{array}{l}4 d^{-2} \\
{ }^{3} F_{4}\end{array}$ & $\begin{array}{l}4 d^{-1} 5 p^{-2}\left({ }^{3} \mathrm{P}\right) \\
{ }^{2} \mathrm{~F}_{5 / 2}\end{array}$ & 26.5 & 26.5 & 26.4 & 26.5 \\
\hline $\begin{array}{l}4 d^{-2} \\
{ }^{1} D_{2}\end{array}$ & $\begin{array}{l}4 d^{-1} 5 p^{-2}\left({ }^{1} S\right) \\
{ }^{2} D_{3 / 2}\end{array}$ & 26.1 & 26.2 & 26.1 & \\
\hline $\begin{array}{l}{ }^{4 d^{-2}} \\
{ }^{1} G_{4}\end{array}$ & $\begin{array}{l}4 d^{-1} 5 p^{-2}\left({ }^{1} S\right) \\
{ }^{2} D_{3 / 2}\end{array}$ & 26.1 & 26.2 & 26.1 & \\
\hline $\begin{array}{l}4 d^{-2} \\
{ }^{3} \mathrm{P}_{2}\end{array}$ & $\begin{array}{l}4 d^{-1} 5 p^{-2}\left({ }^{1} S\right) \\
{ }^{2} D_{5 / 2}\end{array}$ & 25.9 & 26.0 & 25.9 & \\
\hline $\begin{array}{l}4 d^{-2} \\
{ }^{3} F_{4}\end{array}$ & $\begin{array}{l}4 d^{-1} 5 p^{-2}\left({ }^{1} D\right) \\
{ }^{2} G_{9 / 2}\end{array}$ & 25.6 & 25.9 & 25.8 & \\
\hline $\begin{array}{l}4 d^{-2} \\
{ }^{3} F_{2}\end{array}$ & $\begin{array}{l}4 d^{-1} 5 p^{-2}\left({ }^{1} S\right) \\
{ }^{2} D_{5 / 2}\end{array}$ & 24.3 & 24.6 & 24.6 & 24.7 \\
\hline $\begin{array}{l}4^{-2} \\
{ }^{3} \mathrm{~F}_{4}\end{array}$ & $\begin{array}{l}4 d^{-1} 5 p^{-2}\left({ }^{1} S\right) \\
{ }^{2} D_{5 / 2}\end{array}$ & 22.5 & 22.9 & 22.8 & \\
\hline
\end{tabular}
(see text) 
The relativistic many-body PT approach provides physically reasonable results in comparison with the fine experimental results due to a considerable extent to more correct accounting for complex inter electron exchange-correlation effects. It is important to note that using more correct gauge-invariant procedure of generating the relativistic orbital bases in the zeroth approximation of the consistent relativistic perturbation theory is directly connected with correctness of accounting for the exchange-correlation effects. The further theoretical progress can be reached by the next refinement of theoretical procedure.

\section{References}

[1] Jonauskas V., Partanen L., Kucas S., Karazjia R., Huttula M., Aksela S., Aksela H., Auger cascade satellites following 3d ionization in xenon. J. Phys.B. Atom.Mol.Opt. Phys. 2003, 36, 44034416.

[2] Efimova E, Chernyshev A, Buyadzhi V., Nikola L,Theoretical Auger spectroscopy of the neon: transition energies and widths. Photoelectronics. 2019. 28, 24-31

[3] Chernyakova, Y., Ignatenko, A., Vitavetskaya, L.A. Sensing the tokamak plasma parameters by means high resolution x-ray theoretical spectroscopy method: new scheme. Sensor Electr. and Microsyst. Techn. 2004, 1, 20-24.

[4] Aglitsky, E., Safronova, U. Spectroscopy of autoionization states of atomic systems. Energoat: Moscow, 1992.

[5] Glushkov, A.V., Khetselius, O.Yu., Svinarenko, A.A., Buyadzhi, V.V., Spectroscopy of autoionization states of heavy atoms and multiply charged ions. TEC: Odessa, 2015.

[6] Buyadzhi, V., Kuznetsova, A., Buyadzhi, A., Ternovsky, E., Tkach, T. Advanced quantum approach in radiative and collisional spectroscopy of multicharged ions in plasmas. Adv. in Quant. Chem. 2019, 78, 171-191.

[7] Glushkov, A., Buyadzhi, V., Svinarenko, A., Ternovsky, E. Advanced relativistic energy approach in electron-collisional spectroscopy of multicharged ions in plasma. Concepts, Methods, Applications of Quantum Systems in Chemistry and Physics (Springer). 2018, 31, 55-69.

[8] Glushkov, A., Ambrosov, S., Prepelitsa, G., Kozlovskaya, V. Auger effect in atoms and solids: Calculation of characteristics of Auger decay in atoms, quasimolecules and solids with apllication to surface composition analysis. Funct. Materials. 2003, 10, 206.

[9] Nikola, L. Resonant Auger spectroscopy of the atoms of inert gases. Photoelectr. 2011, 20, 104.

[10] Khetselius, O.Yu. Quantum Geometry: New approach to quantization of quasistationary states of Dirac equation for superheavy ion and calculating hyperfine structure parameters. Proc. Int. Geometry Center. 2012, 5(3-4), 39-45.

[11] Ivanov, L.N., Ivanova, E.P., Aglitsky, E. Modern trends in the spectroscopy of multicharged ions. Phys. Rep. 1988, 166.

[12] Svinarenko, A., Khetselius, O., Buyadzhi, V., Florko, T., Zaichko, P., Ponomarenko E. Spectroscopy of Rydberg atoms in a Black-body radiation field: Relativistic theory of excitation and ionization. J. Phys.: Conf. Ser. 2014, 548, 012048.

[13] Glushkov A.V., Ivanov, L.N. DC strong-field Stark effect: consistent quantum-mechanical approach. J. Phys. B: At. Mol. Opt. Phys. 1993, 26, L379386.

[14] Glushkov, A. Spectroscopy of atom and nucleus in a strong laser field: Stark effect and multiphoton resonances. J. Phys.: Conf. Ser. 2014, 548, 012020.

[15] Osmekhin, S., Fritzsche, S., GrumGrzhimailo, A.N., Huttula1, M., Aksela, H., Aksela S. Angle-resolved study of the $\operatorname{Ar} 2 \mathrm{p}^{-1}{ }_{1 / 2} 3 \mathrm{~d}$ resonant $\mathrm{Au}-$ ger decay. J. Phys. B: At. Phys. 2008, 41, 145003.

[16] Pahler, M., Caldwell, C., Schaphorst, S., Krause, M. Intrinsic linewidths of 
neon $2 \mathrm{~s} 2 \mathrm{p}^{5}\left({ }^{1,3} \mathrm{P}\right) \mathrm{nl}^{2} \mathrm{~L}$ correlation satellites. J. Phys. B. At. Phys. 1993, 26, 1617-1622.

[17] Sinanis, C., Aspromallis, G., Nicolaides, C. Electron correlation in Auger spectra of the $\mathrm{Ne}^{+} \mathrm{K} 2 \mathrm{~s} 2 \mathrm{p}^{5}\left({ }^{3,1} \mathrm{P}^{0}\right) 3 \mathrm{p}^{2} \mathrm{~S}$ satellites. J. Phys. B. At. Phys. 1995, 28, L423-428.

[18] Armen, G.B., Larkins, F.P. Valence Auger and X-ray participator and spectator processes for neon and argon atoms. J. Phys. B. At. Mol. Opt. Phys. 1991, 24, 741-760.

[19] Glushkov, A.V. Relativistic and correlation effects in spectra of atomic systems. Astroprint: Odessa, 2006.

[20] Khetselius, O.Yu. Quantum structure of electroweak interaction in heavy finite Fermi-systems. Astroprint: Odessa, 2011.

[21] Glushkov A, Spectroscopy of cooperative muon-gamma-nuclear processes: Energy and spectral parameters $J$. Phys.: Conf. Ser. 2012, 397, 012011.

[22] Khetselius, O.Yu. Hyperfine structure of atomic spectra.-Odessa: Astroprint, 2008.

[23] Glushkov, A.V., Ivanov, L.N. Radiation decay of atomic states: atomic residue polarization and gauge noninvariant contributions. Phys. Lett. A 1992, 170, 33.

[24] Glushkov, A.V.; Ivanov, L.N. DC strong-field Stark effect: consistent quantum-mechanical approach. J. Phys. B: At. Mol. Opt. Phys. 1993, 26, L379386.

[25] Ivanova, E., Glushkov, A. Theoretical investigation of spectra of multicharged ions of F-like and Ne-like isoelectronic sequences. J. Quant. Spectr. and Rad. Tr. 1986, 36(2), 127-145.

[26] Ivanova, E.P., Ivanov, L.N., Glushkov, A., Kramida, A. High order corrections in the relativistic perturbation theory with the model zeroth approximation, Mg-Like and Ne-Like Ions. Phys. Scripta 1985, 32, 513-522.

[27] Dubrovskaya, Yu., Khetselius, O.Yu., Vitavetskaya, L., Ternovsky, V., Serga,
I. Quantum chemistry and spectroscopy of pionic atomic systems with accounting for relativistic, radiative, and strong interaction effects. Adv. Quantum Chem. 2019, 78, 193-222.

[28] Khetselius, O.Yu., Glushkov, A.V., Dubrovskaya, Yu., Chernyakova, Yu., Ignatenko, A., Serga, I., Vitavetskaya, L. Relativistic quantum chemistry and spectroscopy of exotic atomic systems with accounting for strong interaction effects. In: Concepts, Methods and Applications of Quantum Systems in Chem. and Phys. Springer. 2018, 31, 71.

[29] Khetselius, O. Relativistic perturbation theory calculation of the hyperfine structure parameters for some heavy-element isotopes. Int. J. Quant. Chem. 2009, 109, 3330-3335.

[30] Buyadzhi, V.V., Chernyakova, Yu.G., Antoshkina, O., Tkach, T. Spectroscopy of multicharged ions in plasmas: Oscillator strengths of $\mathrm{Be}-$ like ion Fe. Photoelectronics. 2017, 26, 94-102.

[31] Malinovskaya, S., Dubrovskaya, Yu., Zelentzova, T. The atomic chemical environment effect on the $b$ decay probabilities: Relativistic calculation. Herald of Kiev Nat. Univ. Ser.: Phys.Math. 2004, N4, 427-432.

[32] Bystryantseva, A., Khetselius, O.Yu., Dubrovskaya, Yu., Vitavetskaya, L.A., Berestenko, A.G. Relativistic theory of spectra of heavy pionic atomic systems with account of strong pion-nuclear interaction effects: ${ }^{93} \mathrm{Nb},{ }^{173} \mathrm{Yb},{ }^{181} \mathrm{Ta},{ }^{197} \mathrm{Au}$. Photoelectronics. 2016, 25, 56-61.

[33] Buyadzhi, V., Zaichko, P., Antoshkina, O., Kulakli, T., Prepelitsa, P., Ternovsky, V., Mansarliysky, V. Computing of radiation parameters for atoms and multicharged ions within relativistic energy approach: Advanced Code. J. Phys.: Conf. Ser. 2017, 905(1), 012003.

[34] Khetselius, O.Yu., Lopatkin, Yu.M., Dubrovskaya, Yu.V, Svinarenko, A.A. Sensing hyperfine-structure, electro- 
weak interaction and parity nonconservation effect in heavy atoms and nuclei: New nuclear-QED approach. Sensor Electr. and Microsyst. Techn. 2010, 7(2), 11-19.

[35] Danilov, V., Kruglyak, Y., Pechenaya, V. The electron density-bond order matrix and the spin density in the restricted CI method. Theor. Chim. Act. 1969, 13(4), 288-296.

[36] Kruglyak, Yu. Configuration interaction in the second quantization representation: basics with application up to full CI. Science Rise. 2014, 4(2), 98-115.
[37] Glushkov, A.V., Khetselius, O.Yu., Svinarenko, A., Buyadzhi, V. Methods of computational mathematics and mathe-matical physics. TES: Odessa, 2015.

[38] Ignatenko, A.V., Svinarenko, A.A., Prepelitsa, G.P., Perelygina, T.B. Optical bi-stability effect for multiphoton absorption in atomic ensembles in a strong laser field. Photoelectronics. 2009, 18, 103-105.

[39] De Fanis, A., Tamenori, Y., Kitajima, M., Tanaka, H., Ueda, K. Doopler-free resonant Auger Raman spectroscopy on atoms and molecules at Spring-8. J. Phys.: Conf. Ser. 2004, 183, 63-72.

PACS 31.15.A-; 32.30.-r

Chernyshev A.S., Efimova E.A., Buyadzhi V.V., Nikola L.V.

\section{CASCADE OF AUGER TRANSITIONS IN SPECTRUM OF XENON: THEORETICAL DATA}

Summary. The energy parameters of the Auger transitions for the xenon atomic system are calculated within the combined relativistic energy approach and relativistic many-body perturbation theory with the zeroth order density functional approximation. The results are compared with reported experimental data as well as with those obtained by semiempirical methods. The important point is linked with an accurate accounting for the complex exchange-correlation (polarization) effect contributions and using the optimized onequasiparticle representation in the relativistic many-body perturbation theory zeroth order that significantly provides a physically reasonable agreement between theory and experiment.

Key words: relativistic theory, Auger spectroscopy, xenon 
PACS 31.15.A-; 32.30.-r

Чернышев А.С., Ефимова Е.А., Буяджи В.В.. Никола Л.В.

\section{КАСКАД ОЖЕ-ПЕРЕХОДОВ В СПЕКТРЕ КСЕНОНА: ТЕОРЕТИЧЕСКИЕ ДАННЫЕ}

Резюме. Энергетические параметры оже-переходов для атомной системы ксенона рассчитаны в рамках комбинированного релятивистского энергетического подхода и релятивистской многочастичной теории возмущений с приближением функционала плотности нулевого порядка. Результаты сравниваются с экспериментальными результатами, а также с полуэмпирическими теоретическими данными. Важный момент связан с учетом вкладов сложных многочастичных обменных корреляционных 'ффектов и использованием оптимизированного одноквазичастичного представления в нулевом приближении многочастичной теории возмущений, что определяет физически разумное согласие между теорией и экспериментом.

Ключевые слова: релятивистская теория, Оже-спектроскопия, ксенон

PACS 31.15.A-; 32.30.-r

Чернишев О.С., Ефимова С.О., Буяджи В.В.. Нікола Л.В.

\section{КАСКАД ОЖЕ-ПЕРЕХОДІВ В СПЕКТРІ КСЕНОНА: ТЕОРЕТИЧНІ ДАНІ}

Резюме. Енергетичні параметри оже-переходів для атомної системи ксенону обчислені на основі комбінованого релятивістського енергетичного підходу i релятивістської багаточастинкової теорії збурень 3 наближенням функціонала щільності нульового порядку. Результати порівнюються 3 експериментальними результатами, а також з напівемпіричними дтеоретичними даними. Важливий момент пов'язаний з урахуванням вкладів складних багаточасткових обмінних кореляційних ефектів та з використанням оптимізованого одноквазічастічного уявлення в нульовому наближенні релятивістської багаточастинкової теорії збурень, що визначає фізично певну згоду між теорією і експериментом.

Ключові слова: релятивістська теорія, Оже-спектроскопія, ксеон. 